\title{
Mathematical and Computational Modelling in Critical Illness
}

\author{
Marianna Laviola, ${ }^{1}$ Declan G Bates ${ }^{2}$ and Jonathan G Hardman ${ }^{1,3}$ \\ 1. Anaesthesia and Critical Care, Division of Clinical Neuroscience, School of Medicine, University of Nottingham, UK; \\ 2. School of Engineering, University of Warwick, UK; 3. Nottingham University Hospitals NHS Trust, Nottingham, UK
}

DOl: https://doi.org/10.17925/ERPD.2019.5.1.12

$\mathrm{M}$ athematical and computational modelling are assuming a prominent role in investigating the complex pathophysiological states and therapeutic strategies in critical illness. This editorial briefly illustrates the models of the respiratory and cardiovascular systems developed in the last decades, and their advantages and disadvantages with respect to traditional methods of research, i.e. trials in humans and animals. The future direction and application of these high-fidelity and highly integrated models will be to facilitate the development of bedside patient-clones to guide the clinical management of critically ill patients.

\section{Keywords}

Mathematical modelling, computational modelling, critical illness, respiratory system, cardiovascular systems

Disclosure: Marianna Laviola, Declan G Bates and Jonathan G Hardman have no conflicts of interest to declare. Marianna Laviola is a member of the journal's Editorial Board.

Review Process: This article is a short opinion piece and as such, has not undergone the journal's standard peer review process.

Compliance with Ethics: This article is an opinion piece and does not report on new clinical data, or any studies with human or animal subjects performed by any of the authors.

Authorship: The named authors meet the International Committee of Medical Journal Editors (ICMJE) criteria for authorship of this manuscript, take responsibility for the integrity of the work as a whole, and have given final approval for the version to be published.

Received: 9 September 2019

Published Online: 30 September 2019

Citation: European Respiratory \&

Pulmonary Diseases. 2019;5(1):12-3

Corresponding Author: Marianna Laviola, Anaesthesia and Critical Care, Division of Clinical Neuroscience,

School of Medicine, University of Nottingham, Nottingham NG7 2UH, UK. E: marianna.laviola@nottingham.ac.uk

Support: The authors thank EPSRC for the grant "Personalised Simulation Technologies for Optimising Treatment in the Intensive Care Unit: Realising Industrial and Medical Applications" (Grant No. EP/P023444/1).
Life-threatening pulmonary illness is one of the most important reasons for admission to an intensive care unit (ICU). Such illnesses frequently require the use of tracheal intubation, deep sedation and mechanical ventilation. Although mechanical ventilation may keep a patient alive (by ensuring adequate gas exchange), it may lead to further lung injury, may contribute to the systemic inflammatory response and may impair cardiac performance; these may lead to further injury to other organs. Despite years of research, a quantitative understanding of the impact and mitigation of these therapeutic strategies in critical illness is lacking. Additionally, when a patient presents to hospital with pulmonary disease, their condition and their response to therapy are unique. This makes distinguishing between lung diseases, classifying severity and predicting disease progression in an individual difficult.

Due to the emergency nature of the ICU (and emergency room) setting, it is often difficult to recruit patients for clinical trials, and it is challenging to assure appropriate matching, blinding, stratification and control of confounders. ${ }^{2}$ Patient and disease cohorts are very heterogeneous, and, therefore, clinical trial data tend to be noisy and yield inconclusive results. Attempts to address these issues using animal models have not been as successful as was hoped, since they often fail to translate to human medicine, due to differences in pathology and physiology between species, and due to the limited realism of the disease models generated.

Mathematical modelling and computer simulation are novel tools to explore pathophysiological states in critical illness; they represent an alternative to traditional methods of research, i.e. trials on human subjects and in animal models. Virtual (in silico) models of patients and disease-pathology are individualised, mechanistic (meaning that it is possible to look inside them), and amendable to thorough validation. Moreover, computational simulations are effectively free of ethical limitations and are low-cost, very flexible and reproducible. Translation into human applications has met with success in a significant number of recent cases. ${ }^{3-6}$ Using mathematical modelling and computer simulation of the respiratory and cardiovascular systems, researchers can better understand pathophysiological mechanisms in diverse scenarios and can investigate extreme pathophysiology that would otherwise be impossible to study. Investigators can test new hypotheses, optimise patient stratification and inclusion and exclusion criteria, predict the effects of particular treatments or clinical manoeuvres and optimise and personalise physical treatment in critically ill patients.

In recent decades, several models of the respiratory and cardiovascular systems have been developed, with different purposes (i.e., educational or clinical) and various degrees of complexity. The simplest mathematical models of the lungs include a single 'alveolar' compartment representing the whole lung, with a 'balloon-on-a-straw' tidal (or even continuous) ventilation model, and with conducting and respiratory airways and the circulation included in the model.? In such models, there is no change in alveolar and blood volume and they have been mostly used to teach pulmonary physiology.

More recently, high-fidelity, highly integrated models of the pulmonary and cardiovascular systems have been developed with the aim of accurately describing physiological and pathological 
mechanisms, and investigating individualised responses to a pathology or to physical treatment. ${ }^{8-13}$

Limitations of studies performed using mathematical and computational modelling are related to the fact the virtual subjects cannot be configured to match real (human) subjects exactly; however, blinding and validation assure adequate accuracy of matching between real patients and virtual subjects. Moreover, model behaviour at extremes of human physiology is not always reliable because mathematical equations can fail in replicating behaviours at poorly studied extremes, and validation is challenging. However, there is a lack of alternative methodologies to explore such crises. Among cited reasons for failure of clinical translation and incorporation of such models into clinical devices is the lack of engagement with the clinical community. ${ }^{1}$ Thus, the key to the successful complementary use of computer simulators in clinical scenarios is the adoption of an interdisciplinary and collaborative approach, in order to share knowledge and expertise between clinicians, bioengineers and mathematicians. ${ }^{14}$

In the near future, highly integrated and high-fidelity computational models of the pulmonary and cardiovascular systems will facilitate the development of bedside patient-clones, providing real-time simulation of the internal pathophysiological state of individual patients, allowing unprecedented guidance in their clinical management and even facilitating closed-loop control of interventions. It is vital that we equip ourselves with the appropriate skills to use such modelling, and to interpret the results of research based in such methodologies. $\square$
1. Clark AR, Kumar H, Burrowes K. Capturing complexity in pulmonary system modelling. Proc Inst Mech Eng $H$. 2017;231:355-68.

2. Laviola M, Das A, Chikhani M, et al. Computer simulation clarifies mechanisms of carbon dioxide clearance during apnoea. Br J Anaesth. 2019;122:395-401.

3. Das A, Camporota L, Hardman JG, Bates DG. What links ventilator driving pressure with survival in the acute respiratory distress syndrome? A computational study. Respir Res. 2019;20:29.

4. Saffaran S, Das A, Hardman JG, et al. Development and validation of a computational simulator for pediatric acute respiratory distress syndrome patients. Conf Proc IEEE Eng Med Biol Soc. 2017:2017:1521-4.

5. Saffaran $S$, Das A, Hardman JG, et al. High-fidelity computationa simulation to refine strategies for lung-protective ventilation in paediatric acute respiratory distress syndrome Intensive Care Med. 2019:45:1055-7.

6. Szlavecz A, Chiew YS, Redmond D, et al. The Clinical Utilisation of Respiratory Elastance Software (CURE Soft): a bedside software for real-time respiratory mechanics monitoring and mechanical ventilation management. Biomed Eng Online. 2014;13:140.

7. Hahn CEW, Farmery AD. Gas exchange modelling: no more gills, please. Br J Anaesth. 2003;91:2-15.

8. Hardman JG, Bedforth NM, Ahmed AB, et al. A physiology simulator: validation of its respiratory components and its ability to predict the patient's response to changes in its ability to prention Br J Anaesth 1998:81:327-32.

2. Das A Gao Z Menon PP, et al. A systems engineering approach to validation of a pulmonary physiology simulator for clinical applications. J R Soc Interface 2011:8:44-55.
10. Wang W Das A, Ali T, et al Can computer simulators accurately represent the pathophysiology of individual COPD patients? intensive Care Med Exp. 2014:2:23.

11. Das $\mathrm{A}$, Cole $\mathrm{O}$, Chikhani $\mathrm{M}$, et al. Evaluation of lung recruitment maneuvers in acute respiratory distress syndrome using computer simulation. Crit Care. 2015;19:8.

12. Ursino M, Magosso E. Acute cardiovascular response to isocapnic hypoxia. I. A mathematical model. Am J Physiol Heart Circ Physiol. 2000;279:H149-65.

13. Albanese $A$, Cheng $L$, Ursino $M$, Chbat NW. An integrated mathematical model of the human cardiopulmonary system: model development. Am J Physiol Heart Circ Physiol. 2016;310: H899-921.

14. Hardman JG, Ross JJ. Modelling: a core technique in anaesthesia and critical care research. $\mathrm{Br}\rfloor$ Anaesth. 2006:97:589-92. 